Cite this: Soft Matter, 2013, 9, 11634

\title{
Resolving the subnanometric structure of ultrathin films of poly(3,4-ethylenedioxythiophene) on steel surfaces: a molecular modeling approach $\uparrow$
}

\begin{abstract}
David Zanuy ${ }^{\star a}$ and Carlos Alemán*ab
Poly(3,4-ethylenedioxythiophene), abbreviated PEDOT, is one of the most outstanding electroactive polymers, whose applications cover a wide range of fields from biomedicine to electronic components. In this work the physicochemical properties of nanoconstructs obtained by growing PEDOT chains over steel surfaces are investigated. The structural features of such nanostructures, which had successfully been used to build supercapacitors, are investigated, revealing that the polymeric phase plays a key role in the charge accumulation process. The ultra-structure organization and dynamics of these constructs are explored by molecular simulations and the results are compared with the available experimental data. The outcome of this investigation has offered a detailed vision of the structural features that lead to an extraordinarily efficient charge accumulation. Atomistic models generated using stochastic methods had provided different distributions of polymer chains over a metallic surface. Molecular dynamics simulations performed on such models have demonstrated that the extraordinary capacity for charge accumulation stems from a thin balance between the electrostatic repulsion that polymer chains exercise to each other and the attraction that the counterions present in the electropolymerization exert on those oxidized PEDOT chains. This situation favors the aggregation of the PEDOT chains mediated by the perchlorate ions, which act over them as inter-chain cement and which leads to regular three dimensional organization of chains, regardless of their initial distribution over the metallic surface. At the same time, such inner proximity of the polymer chains when growing upwards forces the capture of perchlorate ions, acting as accumulated charges that could be further released if the PEDOT oxidation state is externally reversed.
\end{abstract}

\section{Introduction}

21st century demands in most engineering activities, such as electronics, bioengineering or medical sciences, have converged on the nanometric length scale. This new scenario has induced contemporary researchers of materials science to reconsider several chemical compounds, considered for decades to be impractical materials, as the new 'sorcerers stone' for mastering nano-scale manufacturing. Among such chemical species, conducting polymers (hereafter abbreviated as CPs) have become very attractive building blocks, ${ }^{1}$ due to their induced electrochemical activity, which includes the reversibility of oxidation states, their relative ease of production (via chemical

${ }^{a}$ Departament d'Enginyeria Quimica, E. T. S. d'Enginyeria Industrial de Barcelona, Universitat Politècnica de Catalunya, Diagonal 647, Barcelona E-08028, Spain. E-mail: david.zanuy@upc.edu

${ }^{b}$ Center for Research in Nano-Engineering, Universitat Politècnica de Catalunya, Campus Sud, Edifici C', C/Pasqual $i$ Vila s/n, Barcelona E-08028, Spain. E-mail: carlos.aleman@upc.edu

† Electronic supplementary information (ESI) available. See DOI: $10.1039 / \mathrm{c} 3 \mathrm{sm} 52477 \mathrm{a}$ or electrochemical synthetic methods) and their satisfactory environmental stability.

Poly(3,4-ethylenedioxythiophene), abbreviated PEDOT, possesses the majority of those aforementioned properties: very high conductivity (up to $500 \mathrm{~S} \mathrm{~cm}^{-1}$ ), excellent environmental and chemical stability, fast doping-dedoping processes and exceptional biocompatibility. ${ }^{2-4}$ Moreover, PEDOT presents excellent electrochemical properties in terms of electroactivity and electrostability, explaining the high ability of this material to store charge (i.e. electrical energy). ${ }^{5-7}$

Among the almost never-ending potential applications of PEDOT as a part of nanodevices, it can be used to build electrochemical capacitors. These are charge storage devices of high power that exhibit reversibility and longer life cycles than common batteries. The geometrical and structural arrangements of the device parts determine the capacitance storage and its power. Unlike batteries, electrochemical capacitors store their energy in an electrostatic field rather than in chemical form. ${ }^{8-10}$ Electrode materials for ultracapacitors mainly include carbon, transition-metal oxides, and CPs. ${ }^{11}$ The most common capacitor constructs involving CPs consist of symmetric systems, which use the same p-dopable $\mathrm{CP}$ for the two 
electrodes. PEDOT has recently been used as p-doped electrodes in several nanoconstructs, ${ }^{12}$ using an electrolytic environment media with $\mathrm{LiClO}_{4}$. The electropolymerization and simultaneous oxidation of the growing chains drives the perchlorate ions towards the polymer bulk, neutralizing the polymer charge gain. Hence, the presence of perchlorate anions is a clue for both the material organization and the further dynamics of the capacitors. ${ }^{13}$ In previous investigations the doping level of PEDOT films grown over a steel surface was obtained by determining the amount of $\mathrm{ClO}_{4}{ }^{-}$anions imbibed in the polymer bulk using standard ion chromatography. ${ }^{5}$ The results indicated that the number of positive charges supported by each repeat unit of PEDOT obtained by anodic polymerization was $+0.5,{ }^{5}$ which will be the doping degree set for the simulations presented here.

Ultra-thin PEDOT films have been extensively examined by both scanning electron microscopy (SEM) and atomic force microscopy (AFM) ${ }^{12}$ The electrochemical activity and stability of the produced films have been correlated with the structural, morphological and topographical characteristics of the bulk polymer. However, to date, there are details not yet resolved about the electrochemical deposition and growth of those films over metallic surfaces, mainly due to the length scale that the experimental techniques can cover, over a few dozen nanometers. At this order of magnitude the structural and dynamic features that influence both the polymer growth and its microscopic organization have not yet been revealed. No direct evidence could be extracted about the distribution of polymer chains over the metal, the effect of the doping agents on the final organization or the influence of the degree of oxidation of EDOT units on the structure and dynamics of the polymeric film.

This lack of detailed structural information reaches simple items, such as the direction of the polymer growth. Due to the rigid constitution of EDOT repeat units, researchers have assumed that the polymer chains grew perpendicular to the surface direction, although no detailed investigation has focused on this question, despite the great amount of published information about the electrochemical synthesis of PEDOT on metal surfaces. In order to answer this question we compiled our growth measurements previously published $d^{5,7,12,14,15}$ and we combined them with the available information obtained from quantum mechanics calculations: ${ }^{16,17}$ The collected information strongly suggests that PEDOT chains must grow perpendicular to the metallic surface, at least up to polymer chain lengths comprised in the nanometric scale.

Computations done on short oligomers showed that those structures tend to be rigid regardless of the oxidation state. When the conformation between two continuously bound repeat units of EDOT is trans, the aromaticity of the thiophene ring is drastically enhanced..$^{16}$ Moreover, first principle calculations on oligomers up to 12 repeating units of EDOT clearly demonstrated that the most stable electronic structure of p-doped PEDOT favors a quinoid-type structure for the thiophene ring. ${ }^{17}$ Thus, a highly oxidized PEDOT will present a very rigid and linear structure, without much capacity to show the folded forms of the polymer.
Table 1 shows a compilation of thickness measurements for PEDOT films grown on steel as a function of the electropolymerization time. The former magnitude is directly proportional to the polymer chain length while the latter parameter, polymerization time, is directly proportional to the polymer mass deposited on the metallic surface. We had previously demonstrated that under the current generation conditions PEDOT chains were rigid and linear. We had previously observed as well that the polymer density was constant, regardless of the final polymer thickness. ${ }^{5}$ Therefore, if equal amounts of mass are homogenously incorporated to the polymer bulk, the most probable direction of growth has to be perpendicular to the alloy surface.

This work uses different techniques of molecular simulations to address part of the previously stated questions. First, a reduced molecular model that represents the interface between the PEDOT-covered metal surface and the electropolymerization liquid medium is used to probe several initial distributions of polymer chains, from highly regular distributions of anchoring points to very asymmetrical distributions. A Monte Carlo based strategy is used to generate the latter uneven dispositions and the suitability of each organization is checked using molecular dynamics simulations. Our results will demonstrate that when PEDOT chains are large enough to mimic ultra-thin films, their structural organization is mainly driven by a subtle balance between the electronic repulsions between the positively charged chains of PEDOT, and the electrostatic attraction of EDOT repeat units towards the perchlorate ions. Independent of the polymer rooting positions, the final organization of the thin films always favors the compact clustering of the molecules, with the molecular anions stabilizing the EDOT-EDOT inner electrostatic repulsions by acting as bridging cements.

\section{Methods}

\section{Initial molecular models}

Experimentally, PEDOT is grown over the surface of austenitic stainless steels, or 300 series. $^{12}$ This kind of alloy has a characteristic crystalline structure that consists of a face-centered cubic (abbreviated $f c c$ ) crystal lattice for the iron atom positions,

Table 1 Correlation between time of electropolymerization $(\theta)$ and the thickness of PEDOT grown over steel (in $\mathrm{nm}$ ). The linear regression and the coefficient of correlation have been also included ${ }^{a}$

\begin{tabular}{lrl}
$\theta(\mathrm{s})$ & Thickness $(\mathrm{nm})$ & Reference \\
\hline 5 & 31 & 12 \\
10 & 90 & 12 \\
20 & 284 & 14 \\
30 & 360 & 15 \\
40 & 488 & 14 \\
50 & 609 & 14 \\
60 & 730 & 15 \\
100 & 1340 & 7
\end{tabular}

${ }^{a}$ If all data are included: $y=106.3 x-229.9 ; R 2=0.882$. If the last value is not included: $y=119.2 x-106.7 ; R 2=0.992$. 
with a cell parameter of $3.65 \AA$, whereas the minor components are located on punctual interstitial positions that are mostly dependent on the manufacturing company. ${ }^{18}$ In order to present a reliable representation of the steel structure, avoiding the peculiarities of the minor components, the steel surface will be modeled as a uniform three-dimensional organization of frozen iron atoms organized following a fcc lattice. ${ }^{18}$ Despite being a disfavored crystalline organization for the pure metal, the steel electrodes that were experimentally used presented iron atoms settled in such a crystal organization. Our investigation is basically focused on the structure and dynamics of the polymer, whereas the metal surface role is relegated to a physical support for PEDOT growth and its representation as an iron lattice of frozen atoms is an acceptable approach. Therefore, the metal contribution to the simulation will only be a van der Waals separation wall and sustain for the polymer anchoring. The crystallographic plane used as an anchoring base is $\left(\begin{array}{lll}1 & 1 & 1\end{array}\right)$.

A Monte Carlo based strategy was used for building the starting structures that were further submitted to molecular dynamics simulations (details provided in ESI $\dagger$ ). The main differences among the models are the degree of order with respect to the equatorial distribution of chains over the metallic surface. Those models that present elements of symmetry with respect to the surface distribution are further called ORD, those that broke this symmetrical organization without leaving empty patches in the surface are called DOR and, finally, those that presented areas of no polymer growth are named HOL. Polymer chains consist of 40 repeating units of EDOT, with an oxidation level that accounts for a total charge of +1 every two repeating units. It is worth noting that despite the selected chain length, we did not cover the lowest width experimentally reported, $30 \mathrm{~nm}$, which would have implied a simulation box several times bigger than the one used. Our systems are though realistic, since polymerization times below 5 seconds produce PEDOT chains of the chosen length but do not ensure complete coverage of the metal. ${ }^{5} \mathrm{~A}$ total of 138 chains with their counterions were necessary to cover a surface of $104.1 \times 101.4 \AA$ (details provided in ESI $\dagger$ ), and at the same time fitting the experimental density of p-doped PEDOT films. ${ }^{5}$ The perchlorate ions were placed within the polymer matrix using an early strategy envisaged to estimate the unoccupied space of any polymeric solid. ${ }^{19}$ The maximum amount of 2760 perchlorate ions were placed in the free space left by the PEDOT chains, i.e. the interstitial zones between the polymer chains. The remaining anions were placed above the PEDOT layer, separating each perchlorate molecule by a minimum radial distance of $5.5 \AA$ to ensure its solvation when the solvent is added.

Finally, each built model was placed in a orthorhombic cell of $104.1 \times 104.1 \times 240 \AA$, in which all PEDOT chains were parallel to the crystallographic direction $c$, which was made to coincide with the Cartesian $z$-axis. Finally, all the remaining free space of this simulation box was filled with acetonitrile molecules from a previously equilibrated simulated solvent box that reproduced the experimental density of this liquid $(0.777 \mathrm{~g}$ $\mathrm{cm}^{-3}$ ). Before starting the relaxation and equilibration of each of the initial models, the number of acetonitrile molecules was equalized for all the models, with a total of 21627 solvent molecules (Fig. 1 shows a transversal section of one of the simulated models).

\section{Force field details}

All parameters were extrapolated from AMBER03 libraries, ${ }^{20}$ with the exception of the partial charges of the EDOT repeating unit, which had previously been parametrized. ${ }^{21,22}$ Acetonitrile molecules were represented by the standard AMBER03 model, ${ }^{20}$ and $\mathrm{ClO}_{4}{ }^{-}$parameters were obtain from Baaden et al., 2000. ${ }^{23}$ Bond and length distances of the PEDOT repeat units were approximately kept at their equilibrium distances using the same strategy employed in ref. 22. After an extended bibliographic search, no AMBER compatible parameters were found for iron atoms when organized in an fcc lattice. Since the surface was to be kept fixed in our simulations, we extrapolated the latest published parameters for pure iron in a bcc arrangement to the geometrical features of an fcc arrangement. Ref. 24 shows that solid iron adopting a bcc arrangement presents a cell parameter of $2.87 \AA$ and a metallic radius of $r=$ $1.24 \AA$. Under such a geometrical arrangement the authors presented optimized van der Waals parameters of $\sigma=2.96 \AA$ and $\varepsilon=0.945$. If we represent the investigated steel as an iron material organized following an fcc lattice with a cell parameter of $3.65 \AA$, the apparent metal radius of the iron particles would be $r=1.29 \AA$. If the AMBER parameters of a bcc cell are re-scaled to fit the increment in the atomic radius due to the new lattice structure, the corresponding van der Waals radius is $\sigma=3.08 \AA$.

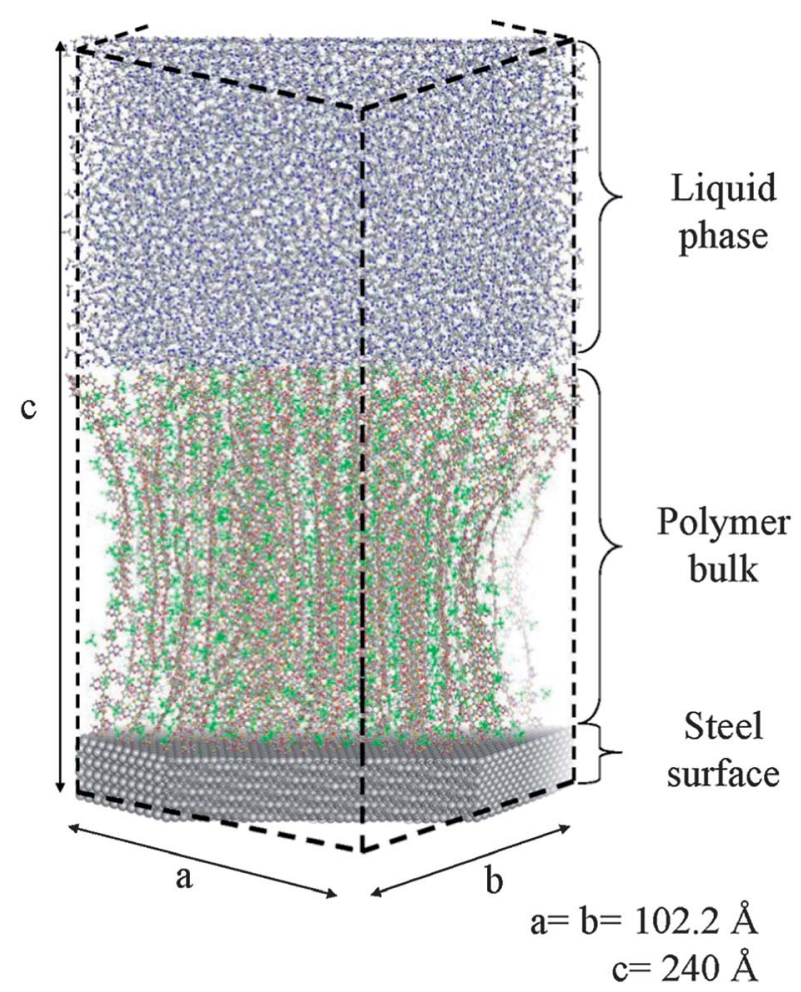

Fig. 1 Transversal cut of the simulation box used for the molecular dynamics simulations of those models previously built using an MC approach. The multiphase nature of the used molecular model is explicitly depicted, including the initial dimensions of the simulated systems. 
For this work, the atomic hardness $(\varepsilon)$ will be kept to the reported value, ${ }^{22}$ because we will not simulate any physical process that implies the active participation of the iron surface. This part of the system will only serve as the anchoring lattice for PEDOT chains.

Periodic boundary conditions were applied using the nearest image convention, and the atom pair cut-off distance was set at 14.0 $\AA$ to compute the van der Waals interactions. In order to avoid discontinuities in the potential energy function, nonbonding energy terms were forced to slowly converge to zero, by applying a smoothing factor from a distance of $12.0 \AA$ A. Beyond the cut off distance, the electrostatic interactions were calculated by using Particle Mesh of Ewald, with a points grid density of the reciprocal space of $1 \AA^{3} .^{25}$

\section{Molecular dynamics: simulation details}

All simulations were performed using the NAMD 2.7 program. $^{26}$ Each system was submitted to 5000 steps of energy minimization (Newton-Raphson method) and this was the starting point of several minicycles of optimization and equilibration. The numerical integration step for all performed runs was set at $1 \mathrm{fs}$, periodic boundary conditions were applied using the nearest image convention and the non bonded pair list was updated every 1000 steps (1 ps).

The first equilibration target was the liquid phase and its ionized interface (i.e. the $\mathrm{ClO}_{4}{ }^{-}$ions present in the solvent phase, see above). This stage was integrated by 100000 steps of heating and equilibration in NVT conditions to thermally stabilize each model at $298 \mathrm{~K}$. The Berendsen thermostat was used $^{27}$ with a relaxation time of $1 \mathrm{ps}$, and the fast convergence of the external bath was used to improve the efficiency of the thermal equilibration. During this period of time all the solid phase atoms were kept frozen.

After that cycle, the solvent density was equalized to its optimum value using 250000 steps of NPT simulation at $298 \mathrm{~K}$. The Nose-Hoover ${ }^{28}$ piston combined with the piston fluctuation control of temperature implemented for Langevin dynamics ${ }^{29}$ was used in this equilibration cycle. Pressure was kept at 1.01325 bars, the oscillation period was set at 1 ps while the piston decay time was set at $0.001 \mathrm{ps}$. The piston temperature was set at the same value as the thermostat control, $298 \mathrm{~K}$, which used a damping coefficient of 2 ps.

Once the simulation box dimensions were stabilized, the final equilibration cycles started. After unfreezing all the atoms present in the solid except for the iron atoms, 200000 more steps of NVT simulation were performed to thermally equilibrate the whole biphasic system. For the latter run the Langevin method $^{30}$ was used to maintain the system temperature constant with a damping coefficient of 2 ps. Finally, 250000 steps of anisotropic pressure control (NPzzT conditions) were run to reach the required simulation conditions. In the NPzzT ensemble, only the box length in the $z$ direction is allowed to change such that the Pzz component of the pressure tensor is equal to the imposed pressure. ${ }^{31-33}$ Thus, in this last mini run, the metallic surface dimensions previously optimized were kept and only the $z$-axis dimension was allowed to change as a response to pressure control. Therefore, the accumulated equilibration time is $0.8 \mathrm{~ns}, 0.25 \mathrm{~ns}$ of which corresponded to the final anisotropic equilibration. The last snapshot of this latter run was the starting point of $15 \mathrm{~ns}$ of production time. The production runs were performed under the same conditions previously mentioned for the NPzzT equilibration.

\section{SEM studies}

PEDOT was polymerized by chronoamperometry under a constant potential of $1.40 \mathrm{~V}$ using a VersaStat II potentiostatgalvanostat connected to a computer controlled through a Power Suite Princeton Applied Research program. Electropolymerization was conducted in a three-electrode twocompartment cell under a nitrogen atmosphere $(99.995 \%$ in purity) at $25{ }^{\circ} \mathrm{C}$. The anodic compartment was filled with $40 \mathrm{~mL}$ of a $10 \mathrm{mM}$ repeating unit solution in acetonitrile containing $0.1 \mathrm{M} \mathrm{LiClO}_{4}$ as a supporting electrolyte, while the cathodic compartment was filled with $10 \mathrm{~mL}$ of the same electrolyte solution. Steel AISI $316 \mathrm{~L}$ sheets of $1 \times 1$ were employed as the working and counter electrodes for generation of the polymer. Films were obtained using a polymerization time of only $10 \mathrm{~s}$. The reference electrode was an $\mathrm{Ag} \mid \mathrm{AgCl}$ electrode containing a $\mathrm{KCl}$ saturated aqueous solution $\left(E^{\circ}=0.222 \mathrm{~V}\right.$ at $\left.25{ }^{\circ} \mathrm{C}\right)$, which was connected to the working compartment through a salt bridge containing the electrolyte solution.

Dried samples were place in a focussed Ion Bean Zeiss Neon 40 scanning electron microscope at $3.00 \mathrm{kV}$.

\section{Results and discussion}

Out of 15 initial structures only the best models representing each general organization (ordered, disordered and with empty patches) were selected. The selection was made on the accounts of total energy and convergence towards a steady organization. From now on, these specific models will be referred as "ORD", "DOR" and "HOL" for the ordered, disordered and patched organizations, respectively (see $\mathrm{ESI} \dagger$ for details).

Fig. 2a shows the time evolution of the mean end to end distance for each studied model. This magnitude has been computed as the average of all end to end distances, over the total number of PEDOT chains, at any given time frame. In all studied cases, the changes in this magnitude point to a quick loss of the polymer original rigidity and show a fairly fast convergence to a more flexible structure. Visual inspection will confirm that in all those cases PEDOT chains display a significant degree of bending inwards of their central segments, reflected as a rapid decrease in the mean end to end distance. It is remarkable that the rate at which each model reaches a steady organization of bent chains is inversely proportional to the degree of regular distribution of chains over the metallic surface. Thus, the ORD model (red line) and DOR model (green line) rapidly reach a steady organization, after roughly two nanoseconds, while the HOL model requires a bit more than 3 nanoseconds to reach that same state.

Fig. $2 \mathrm{~b}$ shows a magnification of the former plot, in which the last 200 ps of NPzzT equilibration has been included (left of 


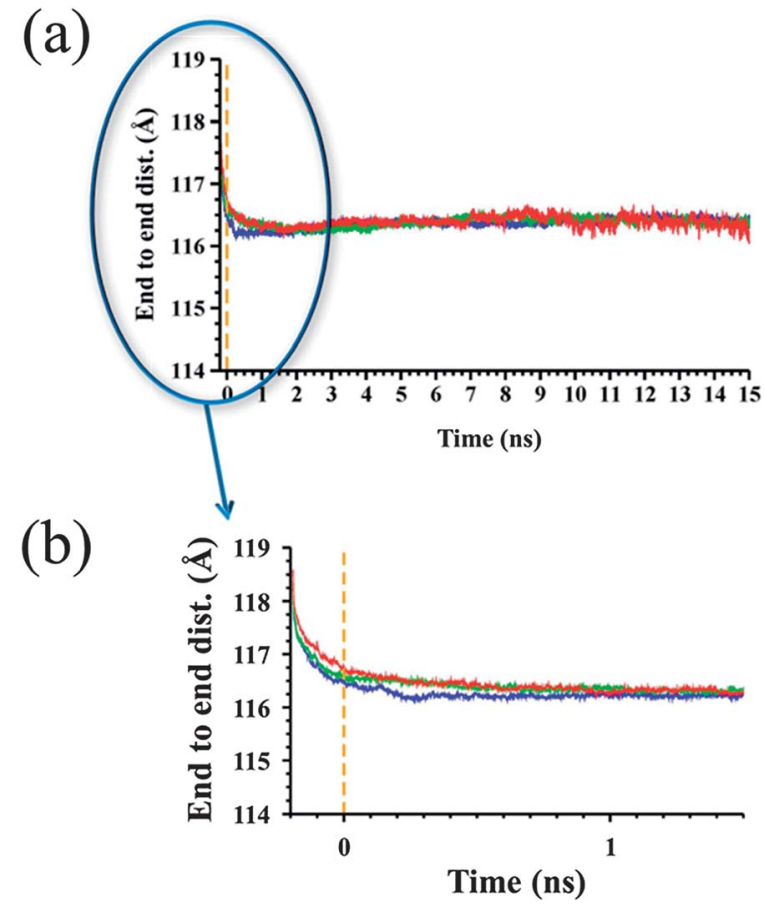

Fig. 2 Temporal evolution of the mean end to end distance of PEDOT chains for each studied model: the ORD (red line), DOR (green line) and HOL (blue line) models, respectively. A dashed orange vertical line separates the equilibration runs from the production ones; (a) evolution over the total simulated time, (b) detail of the mean end to end distance progress for the first $1.7 \mathrm{~ns}$, including a magnified vision of the NPzzT equilibration round.

the dashed vertical line). It is noticeable that the major structural reorganization occurs in that precise short period of time, which demonstrates that the main structural features of PEDOT chains are already present at the first nanoseconds of the production time. This phenomenon can be observed in less than 150 ps of NPzzT equilibration, when the mean end to end distance drops from $118.3 \AA$, $118.6 \AA$ and $118.4 \AA$ to $116.4 \AA$, $116.3 \AA$ and $116.4 \AA$, for the HOL, DOR and ORD models, respectively. In the ESI, $\dagger$ we provide the same plot for all the end to end distances in the DOR model (used an exemplary case that reframes the conclusions made using only the mean distance) and we also provide all the studied averaged values with their standard deviations.

A graphical display of the aforementioned results was obtained by representing the geometrical center of the EDOT repeating units at three different chain positions, a simple approach that emulates a contour lines representation, for all the studied models. Fig. 3 shows that, regardless of the position of the anchor points over the metal (bottom images), the PEDOT chains tend to cluster in the center of the polymer bulk (central images) while the tail edges of the same chains tend to separate from each other. Accordingly, Fig. 3 confirms the final organizations based on a central bending inwards of the polymer chains that seems to aid the formation of compact structures in the central segments of PEDOT, irrespective of the separation between the anchor points or their regular distribution over the metal surface. At this point, and pending deeper analysis, the (a)

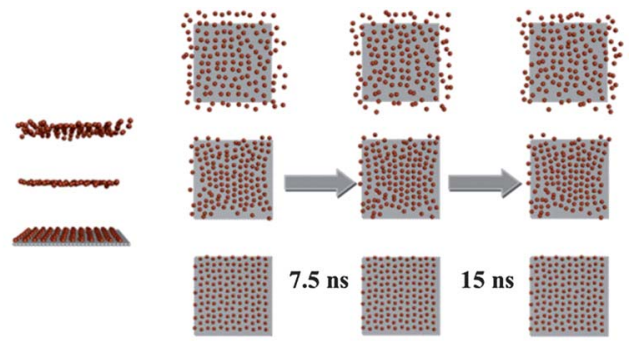

(b)
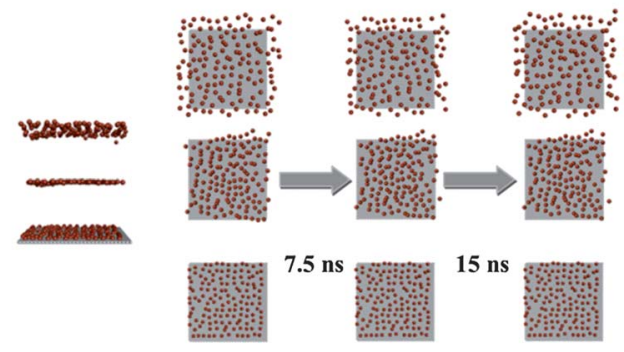

(c)

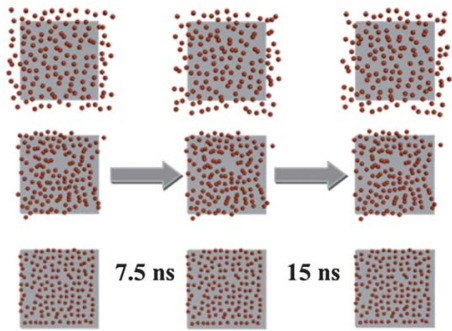

Fig. 3 Equatorial projections of the centers of mass for the EDOT repeating units along the $z$-axis located at 0,50 and $100 \AA$ from the metal surface (see left schemes). Each red sphere represents an EDOT center of mass while the grey surface represents the metallic substrate. The representations depict the evolution of those mass centers at the beginning of the simulation, after $7.5 \mathrm{~ns}$ and 15 ns. From top to bottom: (a) the ORD, (b) DOR and (c) HOL models.

results indicate that the inter-chain organization seems to play a prominent role in the dynamics of PEDOT growing on metal surfaces.

This chain bending, once each system reaches a steady organization, is apparently a common feature of oxidized EDOT oligomers. Previous investigations using short oligomers already revealed this feature and demonstrated that a partial loss of planarity did not affect the electronic properties of the oligomer. ${ }^{34}$ Moreover, recent first principle calculations using longer oligomers, above 20 repeating units of EDOT (in progress $)^{35}$, showed that independent of the degree of oxidation and the electronic state of excitation, EDOT oligomers presented a propensity towards bending, again without loss of its electronic features. Therefore, this is an inner characteristic of EDOT chains, which eventually will be enhanced when the polymer grows in an environment with a high concentration of electrolytes (see below).

In order to obtain further insight into the structural preferences of the polymer phase, the interaction patterns between the polymer chains and the counter-ions have been investigated. The first step was to determine the organization of the polymer chains in terms of intermolecular distances and the variations of these preferred arrangements with time. The radial 
distribution of inter chain distances was computed evaluating the distance between mass centers of individual EDOT repeating units and is shown in Fig. 4. Here, we only show the results for the two most significant profiles, whereas the complete set of results is shown in the ESI, $\dagger$ because the remaining plots do not differ from those shown in Fig. 4. A quick glance reveals two apparently extreme organizations, depending on chain distribution over the metallic surface. The ORD model presents clear differences with respect to the other models, which presenting very similar profiles. For the former model, five peaks stand out over the rest of the inter-chain distances, and these peaks are placed around 7, 8, 11, 13 and 17.5 A respectively.

A visual inspection of representative snapshots of this model confirms that these distances correspond to the most common orientations in which the mass center of any EDOT unit can be
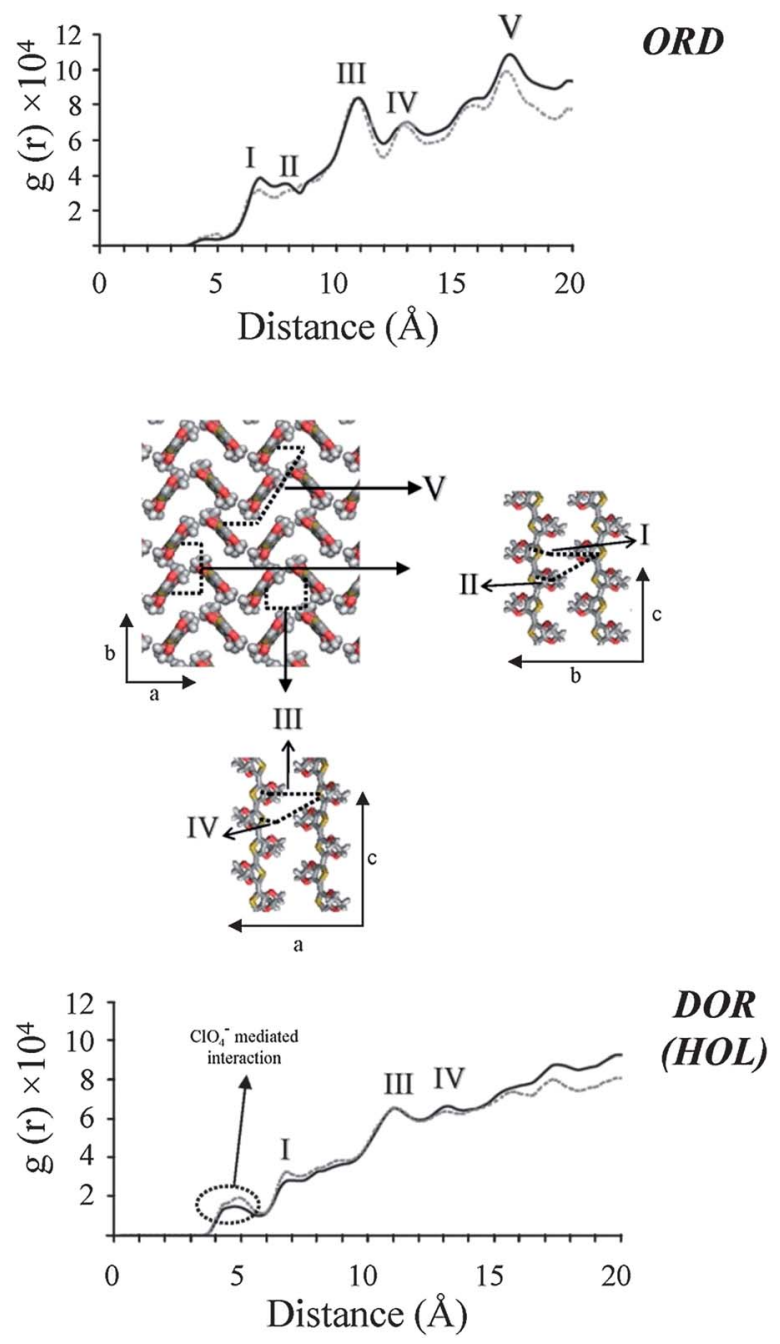

Fig. 4 Accumulated radial distribution of inter chain EDOT-EDOT distances, measured from the centers of mass, for the last ns of simulation. Solid lines depict the results obtained for all polymer chains while dashed grey lines correspond to the central repeating units of each PEDOT chain. Top: plot of the results for the ORD model, middle panel: a schematic representation of the observed structural correlations, and bottom: plot of the results for the HOL model (analogous to those of the DOR model). placed with respect to any neighboring PEDOT chain, when: (i) each neighbor is located at $9.6 \AA$ (distance between any anchor points in the two directions of the metal surface) and (ii) the model presents different setting angles in adjacent layers of chains along the $a$ direction. In the studied systems the phase angle between chains belonging to different layers of PEDOT was $100^{\circ}$. Under such conditions $7 \AA$ and $8 \AA$ are the mass center distances between two EDOT units that belong to different chains on the same layer (see schematic representation in Fig. 4). The former distance corresponds to repeating units in phase (i.e. parallel "stack") while the latter refers to repeating units arranged tail to head (antiparallel "stack"). The following two distances (III and IV in the scheme of Fig. 4) correspond to the same arrangements previously described on chains belonging to different layers. Finally, the last observable peak is again a reflection of the initial organization: if we have layers of chains organized as shown in Fig. S1a of the ESI $\dagger$ and three of those chains are consecutively separated by $9.6 \AA$ and diagonally aligned (see schematic representations in Fig. 4), the distance between the first and the last one will approximately be $17.5 \AA$. The inner organization of the DOR and HOL models, featuring the loss of layered organization, will clearly be responsible for the loss of this peak in those models.

On the other hand, the two remaining models (DOR and $\mathrm{HOL}$ ) share the same distance profile, with a wide ridge around $5 \AA$, a protruding hump between 6.5 and $8.0 \AA$, and the characteristic peaks at 11 and $13 \AA$, also observed in the ORD model. Again these distances are equivalent to the net peaks described for the ORD model, although the higher degree of disorder inherent to the distribution of anchoring points partially diffused the predominance observed in the ORD model. The aforementioned ridge corresponds to a new arrangement closely related to the structural role played by counterions. This new inter-chain EDOT-EDOT distance is the outcome of the self-arrangement performed by the PEDOT chains once the MD was set free. Thus, the positively charged EDOT units during the initial steps of the equilibration tended to repel each other, slightly moving away. This movement creates enough free space "bubbles" to allow the $\mathrm{ClO}_{4}{ }^{-}$anions to penetrate the PEDOT fringes and reverse the repulsion by forming multicentric electrostatic interactions with different neighboring EDOT units, which at last are moved back to each other.

Thus, the dopant anions finally act as temporary cement between polymer chains. It is worth noting that the radial distribution of the ORD model showed an initial behavior that apparently differs from the one observed in both the DOR and HOL models. The explanation stems from its inner regularity, since the even distribution of polymer chains over the metallic surface allowed a more efficient filling of the polymer matrix free space in the stochastic building process (for details see ESI $\dagger$ ), and paradoxically hindered the formation of the proper ionic interactions between the polymer chains. Nonetheless, the appearance of the characteristic signal corresponding to the EDOT-EDOT distances around $5 \AA$ can already be observed after 15 ns of simulation.

The aforementioned topic is confirmed when the dopant anion dynamics is followed with time. Fig. 5 shows the 
distribution of the negatively charged groups inside the polymer bulk at different time frames. The results indicate that only a small amount of $\mathrm{ClO}_{4}{ }^{-}$units remain in the solvent after $5 \mathrm{~ns}$ in the ORD model, because most of them were already in the polymer matrix before heating was performed. In this context, the DOR and HOL models followed analogous paths, filling the maximum possible gaps in their polymer matrices after a short time. Thus, the absorption of the free counterions by the bulk $\mathrm{CP}$ is responsible for the partial reorientation of the PEDOT chains, increasing its compactness in the central segment of the polymer and reducing the impact of the anchoring positions in the final structure and dynamics of the polymer. Accordingly, none of the particular studied cases showed significant differences after the simulated time.

The distribution of $\mathrm{ClO}_{4}{ }^{-}$within the PEDOT bulk has also been examined. The analysis shows that the structural preferences of PEDOT do not affect the structural preferences of the anions inside the polymer matrix. As a matter of fact, time dependent radial distribution of EDOT- $\mathrm{ClO}_{4}{ }^{-}$distances is almost identical for the three studied systems (see ESI $\uparrow$ for more details), revealing three distinct peaks over the smudged distribution for the rest of the computed distances. After visual inspection, such peaks have been attributed to the three most favored modes of interaction between the charged polymer segments and the counterions (Fig. 6). Thus, the peak around 4.5-5.0 A corresponds to the juxtaposition of $\mathrm{ClO}_{4}{ }^{-}$between two chains that are facing each other. The next peak, at $\sim 6 \AA$, corresponds to the same bridging organization where one of the chains is rotated about 90 degrees with respect to the other. The latter peak corresponds to an organization in which the $\mathrm{ClO}_{4}{ }^{-}$ bridges between two chains via the dioxane ring. However, these structural data do not provide any information about the dynamical behavior of the counterions with time, just show that the interaction modes are identical in all studied cases.

The common scenario discussed above indicates that the structural rearrangements suffered in all models converge to a common arrangement that only depends on the balance achieved between the electrostatic interactions of groups of

(a)

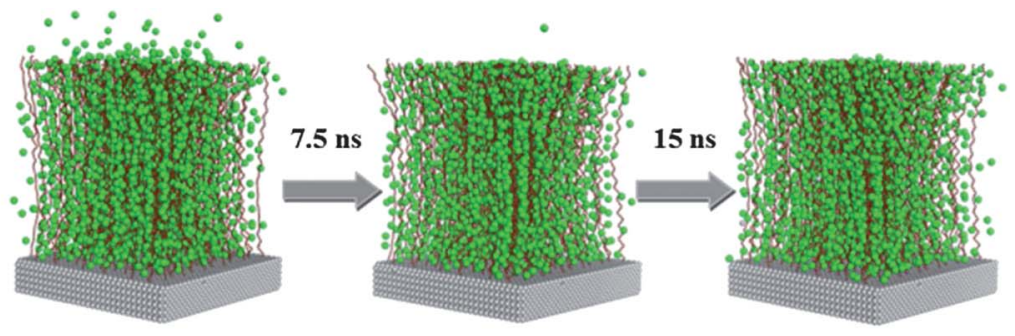

(b)

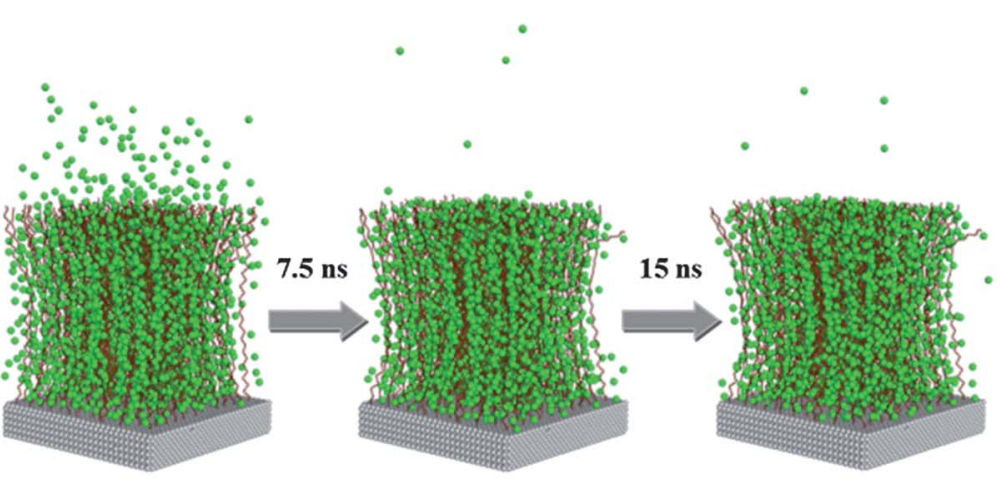

(c)

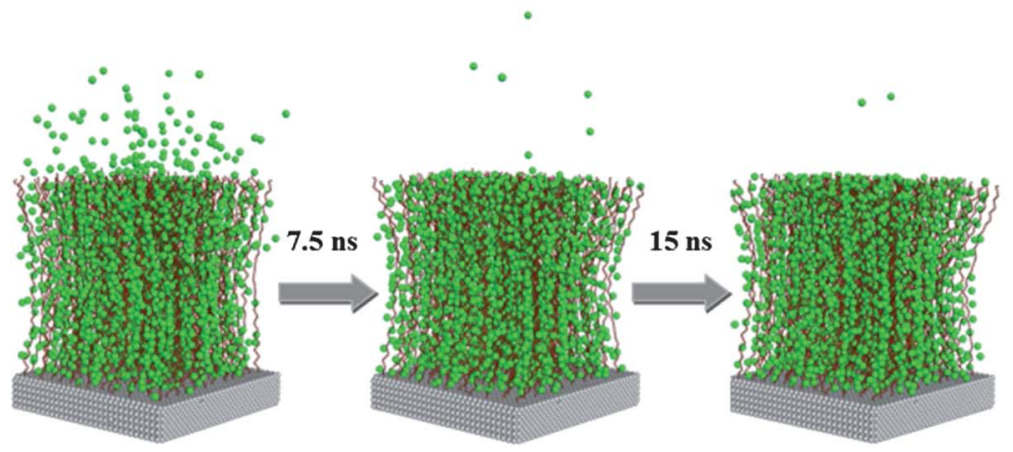

Fig. 5 Distribution of the $\mathrm{ClO}_{4}{ }^{-}$particles within the polymeric matrices at different time frames ( $1 \mathrm{~ns}, 7.5 \mathrm{~ns}$ and $15 \mathrm{~ns}$, respectively) for (a) the ORD, (b) DOR and (c) $\mathrm{HOL}$ models. For clarity, anion particles have been represented as single green spheres (representing their mass centers), PEDOT chains as single backbone chains and the metallic surface as grey spheres. 


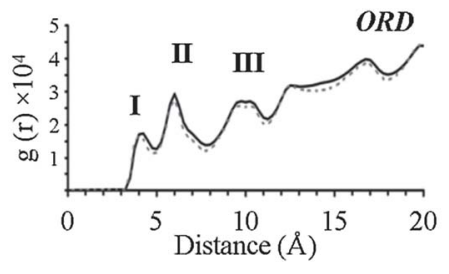

I

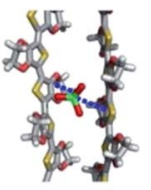

II

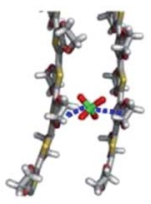

III

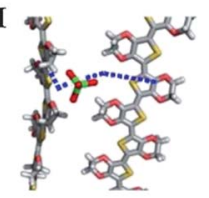

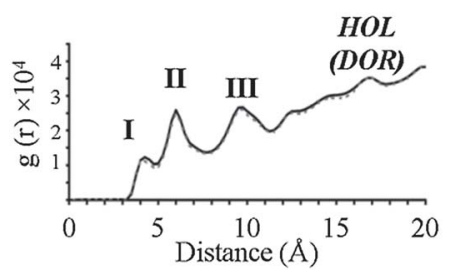

Fig. 6 Accumulated radial distribution of EDOT- $\mathrm{ClO}_{4}{ }^{-}$mass centers distances for the last nanosecond. The solid lines correspond to the distribution obtained using all polymer repeating units and a dashed grey line if only the central ones are studied. Top: the results for the ORD model, middle: a schematic representation of the corresponding structural interpretations, and bottom: the results for the HOL model (analogous to those of the DOR model).

opposite charge sign. As Fig. 5 depicts, counterions penetrate into the polymeric matrix right from the beginning of the simulation, being apparently more efficient in the case of the ORD model. However, this particularity does not differentiate this model from the other two structures since the regular distribution of chains allowed a larger distribution of EDOT- $\mathrm{ClO}_{4}{ }^{-}$distances during the building stage. Due to the chosen strategy for incorporating the anions inside the polymer bulk, ${ }^{19}$ the initial amount of ions in the liquid phase was sensibly lower for the ORD than for the disordered models. Therefore, the counterions sunk in the polymer matrix drive the polymer chains towards a network of lateral interactions. These interactions are mediated by the attraction between the anions and the oxidized repeat units, whereas the inner repulsive forces between the positively charged EDOT units are partially overcome by the previously mentioned partial reorientation of the polymer chains.

Until this point, starting from different initial chains distribution, there has been a rather clear tendency to achieve limited and similar geometrical arrangements of EDOT chains when doped with $\mathrm{ClO}_{4}{ }^{-}$ions. Those interacting patterns determine the final ultra-organization of the PEDOT films. In order to confirm this observation, the energetics of each system was studied, focusing on the polymer matrix behavior. The final aim of this analysis is to evaluate which energy components are the driving forces that lead each studied model to a similar subnano-organization.

In order to obtain a quick assessment, the potential energy of each system has been evaluated. This energy component is expected to represent the largest contribution to the changes in potential energy with time. Under the simulation conditions, in which all the studied models presented the same amount of particles and were run under the same steady conditions of pressure and temperature, changes in the potential energy will be proportional to the major variations of the enthalpy term, while entropic changes would remain similar in all cases, although the ORD model could show discrepancies due its inherent highly ordered organization. Nonetheless, the results presented below will show that our approach suffices to understand the behavior of the simulated material.

The total potential energy of each system at any time step can be expressed as the summation of three independent terms:

$$
E_{\mathrm{tot}}=E^{\mathrm{PEDOT}}+E^{\mathrm{ClO}_{4}^{-}}+E^{\text {acetonitrile }}
$$

where $E^{\mathrm{PEDOT}}$ is the total potential energy of the polymer matrix, $E^{\mathrm{ClO}_{4}^{-}}$is the total energy of the present counterions and $E^{\text {acetonitrile }}$ is the energy of the liquid phase, respectively.

Each contributing term of eqn (1) includes both the potential energy of each component and its interaction with the rest of the system components. Accordingly, each of them can be broken into 3 terms, two of them common to other components (those identified as cross terms) and one proper to each set of species:

$$
E_{\mathrm{tot}}^{\text {species } \mathrm{A}}=E_{\mathrm{A}}+E_{\mathrm{A}-\mathrm{B}}+E_{\mathrm{A}-\mathrm{C}}
$$

where $E_{\mathrm{A}}$ is the internal energy of species $\mathrm{A}$, that includes intramolecular terms and the interaction energy between all $\mathrm{A}$ molecules, and $E_{\mathrm{A}-\mathrm{B}}$ and $E_{\mathrm{A}-\mathrm{C}}$ are the interaction energies cross terms, that are common to in the expression of the total energy of B and C species (if they were to be computed).

For the purposes of the current investigation, the EDOT intramolecular terms are neglected and $E_{\mathrm{A}}$ will be approached to $E_{\mathrm{A}-\mathrm{A}}$ (not shown in eqn (2), since it is part of $E_{\mathrm{A}}$ ), which computes the interaction energy of each EDOT chain with the rest of the bulk chains. Thus the total energy of the PEDOT bulk can be approached to its intermolecular components as:

$$
E_{\mathrm{PEDOT}}=E_{\mathrm{PEDOT}-\mathrm{PEDOT}}+E_{\mathrm{PEDOT}-\mathrm{ClO}_{4^{-}}}+E_{\mathrm{PEDOT}-\text { acetonitrile }}(3)
$$

where $E_{\mathrm{PEDOT}-\mathrm{PEDOT}}$ represents the interaction energy between PEDOT chains, and both $E_{\mathrm{PEDOT}-\mathrm{ClO}_{4}-}$ and $E_{\mathrm{PEDOT}-\text { acetonitrile }}$ are the same cross terms shown in eqn (1) and (2).

Preliminary studies over the first $5 \mathrm{~ns}$ of the trajectory proved that the energy of the PEDOT matrix only depended on the changes of the two first components of eqn (1), as can be seen from the data shown in Table 2. The contribution of $E_{\text {PEDOT-acetonitrile }}$ over $E_{\mathrm{PEDOT}}$ is 7 orders of magnitude lower than the remaining components, being totally negligible. These results are not surprising since PEDOT is highly insoluble in acetonitrile, which induces a clear phase separation expressed as very low values of positive interaction energy and nil solubility of acetonitrile molecules within the polymer matrix. It is worth noting that the insolubility of PEDOT in acetonitrile is an experimental requirement for a successful anodic polymerization of EDOT repeating units. Thus, the generated PEDOT 
Table 2 Decomposition in different contribution terms of the PEDOT potential energy over the first 5000 picoseconds of simulation (see eqn (1) and (2)). All data are given in kcal (repeat unit of EDOT) $)^{-1 a}$

\begin{tabular}{lllll}
\hline Model & $E_{\text {PEDOT-PEDOT }}$ & $E_{\text {PEDOT-ClO }_{4}^{-}}$ & $E_{\text {PEDOT-acetonitrile }}$ & $E^{\text {PEDOT }}$ \\
\hline ORD & $2119.8 \pm 29.6$ & $-3915.4 \pm 57.1$ & $-7.5 \pm 0.3 \times 10^{-4}$ & $-1805.1 \pm 27.7$ \\
DOR & $2074.4 \pm 25.7$ & $-3826.2 \pm 37.5$ & $-6.9 \pm 0.6 \times 10^{-4}$ & $-1751.9 \pm 19.4$ \\
HOL & $2074.3 \pm 14.7$ & $-3823.8 \pm 23.1$ & $-8.7 \pm 1 \times 10^{-4}$ & $-1749.4 \pm 19.2$
\end{tabular}

${ }^{a}$ All energy data presented in this work is expressed as kcal per the number of EDOT repeat units.

chains must be electrodeposited onto the electrode to form films but they must not be dissolved in the polymerization medium. Accordingly, the analysis of the full trajectories will not include the $E_{\text {PEDOT-acetonitrile }}$ contribution.

The time-dependent evolution of those two principal energy components and their respective summation can be followed in Fig. 7. The temporal evolution of the $E_{\text {PEDOT-PEDOT }}$ contribution indicates that the higher the inner order of the chains distribution, the lower the stability of the packing (Fig. 7a). Throughout the whole simulation the interaction energy between PEDOT chains always shows significantly more positive values for the ORD model than for the other studied models.

There is though, a simple interpretation to these results. The $E_{\text {PEDOT-PEDOT }}$ contribution will always disfavor the packing of PEDOT chains because of their positively charged segments, which will always imply electrostatic repulsion. Thus, the higher the regularity in the chains distribution through the metal surface, the lower the re-organization margin for PEDOT chains to diminish such energy penalty. It is worth noting that the reorganization of the lateral packing takes place during the equilibration period, which makes invisible the changes on the PEDOT-PEDOT interaction energy as they first highly repel, especially in both the DOR and HOL models. Those latter systems presented a significant amount of $\mathrm{ClO}_{4}{ }^{-}$anions dissolved in the liquid phase that during the equilibration period rapidly diffuse towards the polymer bulk.

On the other hand, Fig. 7b shows that the interaction of PEDOT with $\mathrm{ClO}_{4}{ }^{-}$ions seems to be favored when the polymer matrix is relatively ordered (ORD model). However, this point must be carefully considered, since the initial disposition of ions inside the polymer had not been completed for the disordered models. As we mentioned above, the generation strategy could not complete the insertion of all $\mathrm{ClO}_{4}{ }^{-}$particles due to the uneven distribution of free space inside the polymer phase. Thus, the main differences for the $E_{\mathrm{PEDOT}_{-} \mathrm{ClO}_{4}}$ contribution were mainly due to the unbalanced charges of the latter models, which exhibited some counterions in the acetonitrile phase at the initial steps of the simulations. Once all the ions are inside the polymer matrix in those models, the energy differences were significantly reduced.

The overall results depict an internal structural reorganization in all the studied cases that allows optimization of the electrostatic interactions set by both charged species. Once the repulsions between polymer chains were shielded and overpassed by the electrostatic attraction with the anions that got dissolved into the polymer matrix, the PEDOT bulk becomes energetically favored and a steady and stable state is reached. This new state is then featured by the formation of an interaction network in which perchlorate ions act as the key elements for keeping the polymeric organization once they have reached their equilibrium positions inside the PEDOT moiety.

In summary, the results indicate that the subnanometric organization of the PEDOT film implies a fairly regular distribution of chains over the metallic surface while the spatial disposition along the growth direction gets disorganized, mainly due to both the inner chains bending and the

(a)

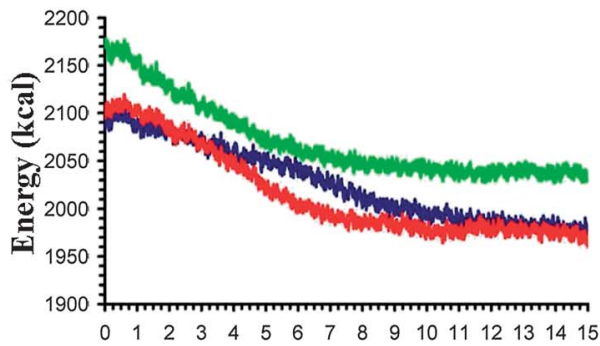

(b) Time (ns)

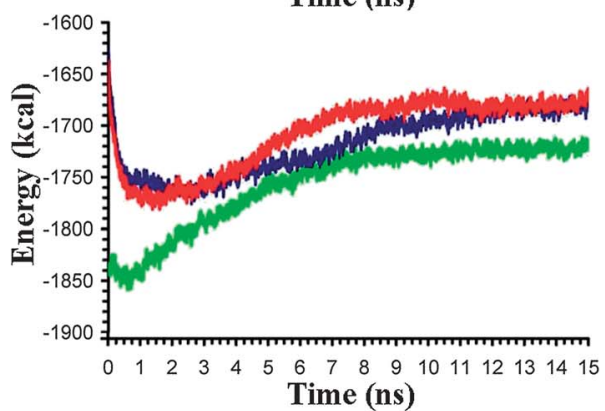

(c)

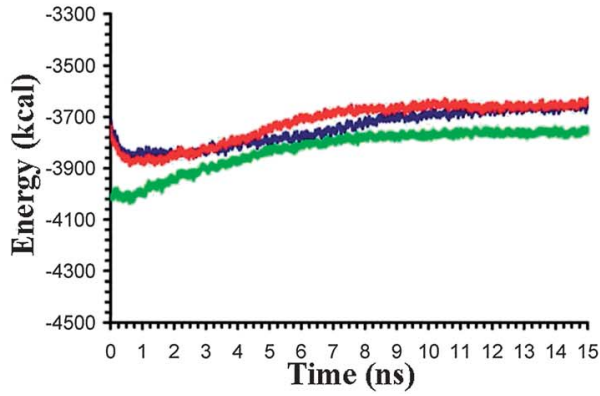

Fig. 7 Temporal evolution of the principal terms of potential energy for the three studied models: (a) $E_{\mathrm{PEDOT}-\mathrm{PEDOT}}$ and (b) $E_{\mathrm{PEDOT}-\mathrm{ClO}_{4^{-}}}$contributions to the interaction energy and (c) summation of those two terms (see text for details). The green line corresponds to the ORD model, the red line to the DOR model and the blue line to the HOL model. All energy components are expressed as kcal (repeat unit of EDOT) ${ }^{-1}$. 
electrostatically mediated clustering of the chains middle and top segments, as seen in all models. This molecular description is fully compatible with experimental observations obtained for films of nanometric thickness (i.e. those produced using very small polymerization times). ${ }^{\mathbf{1 2 , 1 4 3 6}}$ Thus, anodic polymerization of EDOT repeat units using steel or indium tin oxide (ITO) electrodes produces a complete coverage of the substrate, even for short polymerization times (i.e. $\leq 10 \mathrm{~s}) .{ }^{12}$ This feature, which is consistent with the regular distribution of chains over the surface, is accompanied by a clustered organization of the polymer chains. ${ }^{\mathbf{1 2 , 1 4 , 3 6}}$ More specifically, AFM images of films obtained using polymerization times of 5 and $10 \mathrm{~s}$ showed that PEDOT chains deposited on steel surfaces tend to organize forming dense distributions of sharp peaks grouped into clusters. Moreover, comparison of films produced using polymerization times of 5, 10, 30, 50 and $300 \mathrm{~s}$ evidenced that the size and number of these clusters increases and decreases, respectively, with polymerization time. Thus, films obtained using a polymerization time of $5 \mathrm{~s}$ allowed us to identify a large number of clusters, each one occupying a relatively small area, separated by flat regions of very different sizes heterogeneously distributed. This particular organization, which is in agreement with the bending and clustering evidenced by the atomistic MD simulations discussed in this work, has been corroborated by SEM, as is displayed in Fig. 8.

Even though the scale of the SEM images is about 100 times bigger than the studied models scope, the only possible way of growing perpendicularly to the metal surface with such heterogeneous organization is to follow the molecular organization previously presented. On the other hand, PEDOT has been found to be a porous material (Fig. 8), which explains the remarkable ability of this conducting polymer to store charge. ${ }^{7,14}$ Thus, $\mathrm{ClO}_{4}{ }^{-}$ions easily access and escape from the polymeric matrix upon oxidation and reduction processes, respectively. The clustered organization found in this work also can explain the electrochemical behavior of PEDOT, providing an atomistic description of the influence of the dopant ions in the structure of the polymer. This latter item is the main goal of

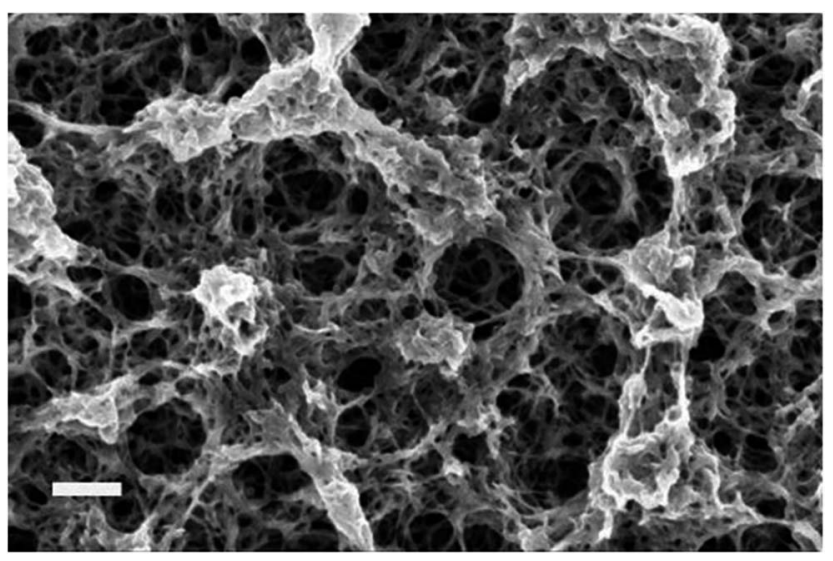

Fig. 8 High magnification (scale bar: $200 \mathrm{~nm}$ ) SEM image of PEDOT electrodeposited on a steel substrate. The film displayed was obtained using a polymerization time of $10 \mathrm{~s}$. the work in progress, which simulates and studies the growing preferences, i.e. the formation of polymer chains of different lengths.

\section{Conclusions}

The investigation of the ultra-structure of novel materials aids the comprehension of the physical properties experimentally observed, whereas it holds the key to the improvement of its practical performance for specific applications. Within this general frame, we have studied the organization of poly $(3,4$ ethylenedioxythiophene) grown over a steel surface, a material that has shown promising applications in electronics as high performance capacitors. Our research has focused on two different levels of organization and has used two different and complementary approaches: an atomistic description of the material based on molecular modeling approaches and its comparison with real structures at the nanometric scale using electron microscopy.

The atomistic view obtained using a combination of model generation using stochastic methods combined with the dynamic vision obtained using molecular dynamics simulations, shows a subnanometric organization that is driven by apparently two different factors that merge into a single determinant cause, an electrostatic balance of repulsion and attractions between the charged groups. Thus, the oxidized PEDOT requires a sufficiently loose distribution of chains to allow the counterions to penetrate into the polymer matrix and avoid the electrostatic repulsion that positively charged chains would undergo. Nonetheless, a high degree of disorganization with respect to the distribution of polymer chains over the metallic surface does not favor polymer growth, which limits as the same time the formation of important voids over the supporting surface. The overall consequence of such a delicate balance drives the formation of clusters of polymer chains that magnify the presence of perchlorate ions between polymer chains, fixing their positions in space as agglutination agents.

This micro-structural organization is fully consistent with the experimental observations that showed large number of polymer clusters, each one occupying a relatively small area, separated by flat regions of very different sizes heterogeneously distributed. At the same time, our molecular models are fully consistent with the electronic properties of the material, since its excellent capacity to integrate counterions inside the polymer matrix is the key factor for its application in supercapacitors, since the charge is directly accumulated as ionic particles absorbed within the polymer bulk. Hence, the stabilization of the ultrastructure adopted by oxidized polymer chains is at the same time responsible for the ability of PEDOT to accumulate charges as a capacitor.

\section{Acknowledgements}

Financial support from MCYT and FEDER (MAT2012-34498), by the Generalitat de Catalunya (research group 2009 SGR 925 and XRQTC) and CESCA is gratefully acknowledged. Support for the research of C.A. was received through the prize "ICREA 
Academia" for excellence in research funded by the Generalitat de Catalunya. Authors are thankful to Dr Aradilla for his kind assistance in SEM studies.

\section{Notes and references}

1 Y. Z. Long, M. M. Li, C. Gu, M. Wanc, J. L. Duvail, Z. Liu and Z. Fan, Prog. Polym. Sci., 2011, 36, 1415-1442.

2 S. Kirchmeyer and K. Reuter, J. Mater. Chem., 2005, 15, 20772088.

3 L. J. del Valle, F. Estrany, E. Armelin, R. Oliver and C. Alemán, Macromol. Biosci., 2008, 8, 1144-1151.

4 M. J. Higgins, P. J. Molino, Z. Yue and G. G. Wallace, Chem. Mater., 2012, 24, 828-839.

5 C. Ocampo, R. Oliver, E. Armelin, C. Aleman and F. Estrany, J. Polym. Res., 2006, 13, 193-200.

6 E. Tamburri, S. Orlanducci, F. Toschi, M. L. Terranova and D. Passeri, Synth. Met., 2009, 159, 406-414.

7 D. Aradilla, F. Estrany and C. Aleman, J. Phys. Chem. C, 2011, 115, 8430-8438.

8 R. Kotz and M. Carlen, Electrochim. Acta, 2000, 45, 2483-2498.

9 E. Frackowiak and F. Beguin, Carbon, 2002, 40, 1775-1787.

10 Y. G. Wang and X. G. Zhang, Electrochim. Acta, 2004, 49, 1957-1962.

11 C. Liu, F. Li, L.-P. Ma and H.-M. Cheng, Adv. Mater., 2010, 22, E28-E82.

12 D. Aradilla, F. Estrany, E. Armelin and C. Alemán, Thin Solid Films, 2012, 520, 4402-4409.

13 D. K. Bhat and M. S. Kumar, J. Mater. Sci., 2007, 42, 81588162.

14 D. Aradilla, F. Estrany, E. Armelin and C. Alemán, Thin Solid Films, 2010, 518, 4203-4210.

15 F. Estrany, D. Aradilla, R. Oliver, E. Armelin and C. Aleman, Eur. Polym. J., 2008, 44, 1323-1330.

16 J. Poater, J. Casanovas, M. Sola and C. Aleman, J. Phys. Chem. A, 2010, 114, 1023-1028.

17 J. Torras, J. Casanovas and C. Alemań, J. Phys. Chem. A, 2012, 116, 7571-7583.

18 M. F. McGuire, Stainless Steels for Design Engineers, ed. J. G. Kaufman, ASM International, 2008, ch. 6, pp. 69-78.

19 D. Curcó, D. Zanuy, D. and C. Alemán, J. Comput. Chem., 2003, 24, 1208-1214.
20 Y. Duan, C. Wu, S. Chowdhury, M. C. Lee, G. Xiong, W. Zhang, R. Yang, P. Cieplak, R. Luo, T. Lee, J. Caldwell, J. Wang and P. Kollman, J. Comput. Chem., 2003, 24, 19992012.

21 B. Teixeira-Dias, D. Zanuy, L. J. del Valle, F. Estrany, E. Armelin and C. Alemán, Macromol. Chem. Phys., 2010, 211, 1117-1126.

22 J. Preat, D. Zanuy, E. A. Perpete and C. Aleman, Biomacromolecules, 2011, 12, 1298-1304.

23 M. Baaden, M. Burgard, C. Boehme and G. Wipff, Phys. Chem. Chem. Phys., 2001, 3, 1317-1322.

24 D. Savio, N. Fillot, P. Vergne and M. Zaccheddu, Tribol. Lett., 2012, 46, 11-22.

25 A. Toukmaji, C. Sagui, J. Board and T. Darden, J. Chem. Phys., 2000, 113, 10913-10927.

26 J. C. Phillips, R. Braun, W. Wang, J. Gumbart, E. Tajkhorshid, E. Villa, C. Chipot, R. D. Skeel, L. Kale and K. Schulten, J. Comput. Chem., 2005, 26, 1781-1802.

27 H. J. C. Berendsen, J. P. M. Postma, W. F. van Gunsteren, A. DiNola and J. R. Haak, J. Chem. Phys., 1984, 81, 36843690 .

28 G. J. Martyna, D. L. Tobia and M. L. Klein, J. Chem. Phys., 1994, 101, 4177-4189.

29 S. E. Feller, Y. Zhang, E. W. Pastor and B. R. Brooks, Chem. Phys., 1995, 103, 4613-4622.

30 S. Toxvaerd, J. Chem. Phys., 1990, 93, 4290-4295.

31 C. L. Wennberg, T. Murtola, B. Hess and E. Lindahl, J. Chem. Theory Comput., 2013, 9, 3527-3537.

32 S. Pandiyan and B. Rousseau, Polymer, 2013, 54, 3586-3593. 33 J. A. Diaz, X. Wu, A. Martini, J. P. Youngblood and R. J. Moon, Biomacromolecules, 2013, 14, 2900-2908.

34 J. Casanovas and C. Alemán, J. Phys. Chem. C, 2007, 111, 4823-4830.

35 Personal communication from Carlos Alemán: all calculations performed on PEDOT oligomers ranging from 20 to 30 repeat units. In all studied cases, geometry optimization of all possible electronic states were computed using the unrestricted DFT formalism UB3LYP/ 6-31G(d). More details are provided in the ESI. $\dagger$

36 D. Aradilla, D. Azambuja, F. Estrany, M. T. Casas, C. A. Ferreira and C. Alemán, J. Mater. Chem., 2012, 22, 13110-13122. 